\title{
THE INFLUENCE OF ROTATIONAI SPEED ON TEMPERATURE RISE DURING CAVITY PREPARATION
}

\author{
R. C. VAUGHN, B.A., AND F. A. PEYTON, D.Sc. \\ Sohool of Dentistry, Eniversity of Michigan, Ann Arbor, Mich.
}

$\mathrm{R}$ ECENTLY there has been considerable interest in the possible use of high speeds and various hand pressures as they influence the temperature rise during cavity preparation. One study has been reported in which speeds up to 60,000 r.p.m. have been used. ${ }^{1}$ A previous report from this laboratory has described thermal changes developed during the cutting of tooth tissue where various instruments are used at speeds produced by the conventional commercial dental engine. ${ }^{2}$ From each of these studies it is apparent that in the use of rotating instruments to cut tooth tissue, the problem of heat generation is only one part of a larger problem which also includes the efficiency, vibration characteristics, and life expectancy of the rotating cutting instrument. Inasmuch as each of these four factors is influenced by several variables such as size, design, speed of operation and pressure applied, it is apparent that the application of the rotating dental instrument to the eutting of tooth tissue is basieally a complex operation.

The purpose of this study was to determine the effect of speeds up to 11,300 r.p.m. on the temperature developed in the tooth during the cutting of enamel and dentin simultaneously. For this study only one design of bur (fissure type) was employed. To a limited degree the force applied during the cutting operation and the size of the instrument was varied. These variations, however, were well within the limits of conventional practice.

\section{METHOD OF TEST}

Extracted noncarious molar teeth were used in all tests described. These teeth were stored in tap water until they were needed for tests. Each tooth was prepared to accommodate a thermocouple by cutting a hole to the dentinenamel junction at a point approximately 1 to $2.5 \mathrm{~mm}$. below the ocelusal surface, using a number one round bur.

A thermocouple of 28 gauge chromel-alumel wires, connected to a Type " $G$ " Leeds and Northrup Speedomax potentiometer-recorder, was wedged into the tooth at the dentino-enamel junction. This connection allowed temperature recordings to be made at four-second intervals.

A beam balance was constructed so that it was supported in the center and could be loaded at either end. One end was designed to hold a platform on which the teeth were mounted in plaster. The other end was counterbalanced by filling a cup with shot, in sufficient quantity to counteract the weight of the

A portion of this study was supported by a grant from the Horace H. Rackham School of Graduate Studies, Project R-217, University of Michigan.

Read at the meeting of the Materials Group International Association for Dental Research, March 17, 1951, French Lick, Ind. (J. D. Res. 30: 497, 1951) .

Received for publication. April 24, 1951. 

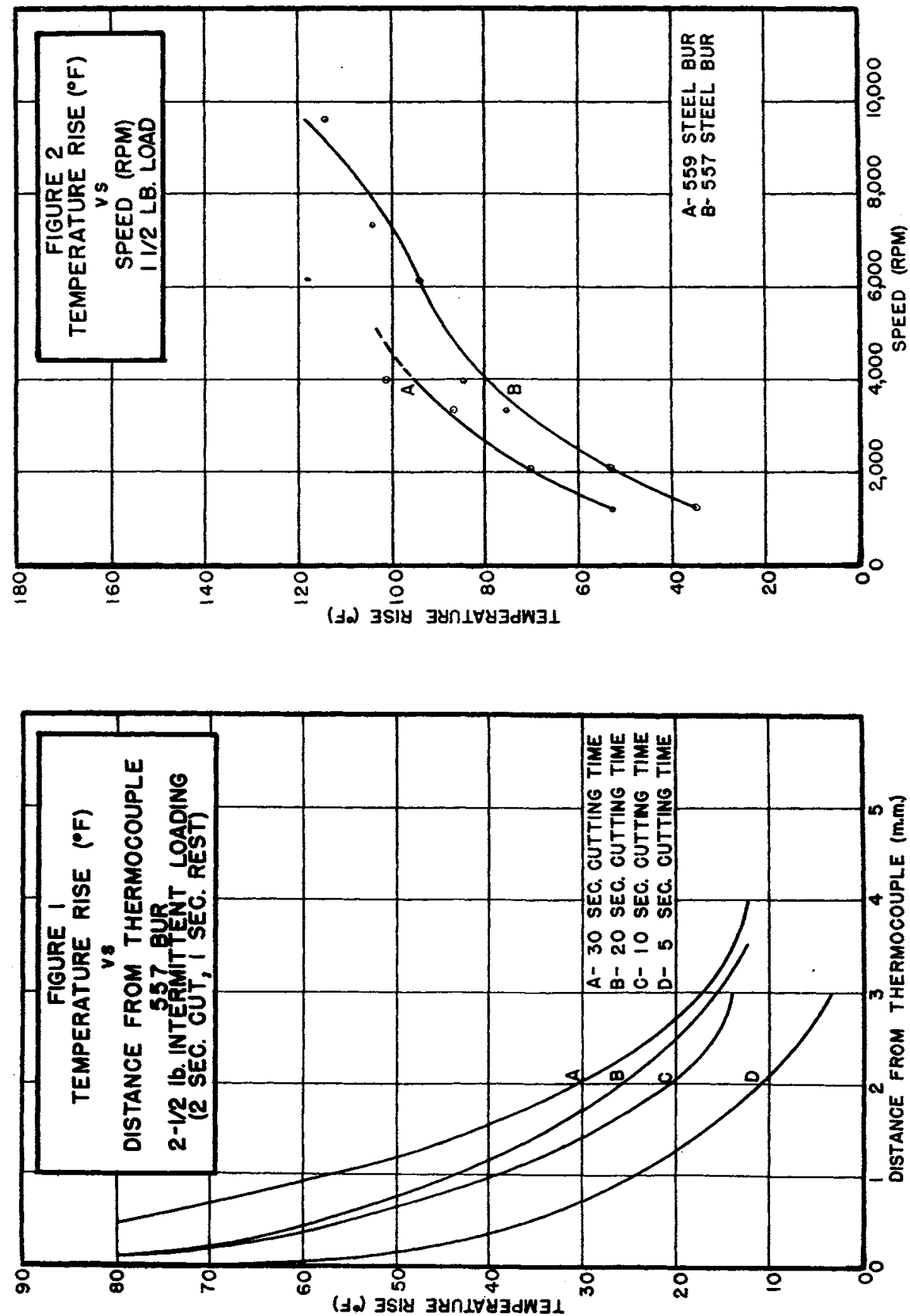
platform, plaster, and tooth. Below this counterbalance were suspended known weights. A support was positioned so that the beam would be level before a load was applied to the tooth during the cutting operation.

A four-speed laboratory motor capable of rotating at speeds of 1,310, 2,200, 3,400 , and 4,000 r.p.m. at the driveshaft was used to operate a conventional dental handpiece. By varying the diameter of the drive pulley, and using the four motor control settings, various speeds between 1,155 and 11,300 r.p.m. were obtainable at the handpiece. To cover the range of rotational speeds available, it was necessary to employ only six or eight operating speeds, such as 1,155 , $2,200,3,400,4,000,6,200,7,300,9,600$, and 11,300 r.p.m.

Except for data involving tungsten carbide burs and spiral fluted burs, new burs of one product, purchased through usual trade sources, were used throughout the tests. A new steel bur was used for each measurement, so that the effect of pronounced dulling would not produce variable results. In the case of tungsten carbide burs, they were used until a microscopic comparison of used and new instruments showed the used ones to be slightly dulled. The spiral flutted steel burs described in this report were of a different make, but no comparison of various trade products is attempted. The fissure bur proved adaptable to laboratory tests for cutting enamel and dentin, and its wide application in dental practice justifies this study in detail. Throughout this report all values listed are averages of at least two, but usually several more, measurements.

\section{DISCUSSION OF RESULTS}

If the temperature rise at the surface being eut is known under a given set of conditions of operation, and if the distance of this surface from the pulp chamber can be estimated with some accuracy, an approximation of the temperature rise at the pulp can be made. For use in such determinations, the temperatures developed at various distances from the thermocouple are shown in Fig. 1, with curves for cutting times of $5,10,20$, and 30 seconds. To obtain the 5,10 , and 20 second temperature vs. distance curves, vertical cuts in tooth structure were made from the occlusal surface of the tooth at various distances from the thermocouple. A load of $2 \frac{1}{2}$ pounds was used on a No. 557 steel bur for these tests. The operating speed was 3,400 r.p.m. Data for the 30 second cutting interval were obtained as described previously. ${ }^{2}$ From these curves, it is apparent that near the heat source a large temperature rise is obtained soon after the operation begins.

Throughout the study, intermittent cutting was used. The time cycle for this was two seconds of cutting followed by one second rest. While this procedure may allow slightly greater variation in the results than if the eutting is constant, it was thought advisable to use a method which closely resembled that usually recommended in practice. The cutting time was not rigidly controlled, except for results deseribed in Fig. 1, but the thermocouple was placed at a position such that approximately two minutes elapsed before the thermocouple was reached. The temperatures recorded were those obtained when cutting within $0.5 \mathrm{~mm}$. of the thermocouple. All temperature rises were measured from 

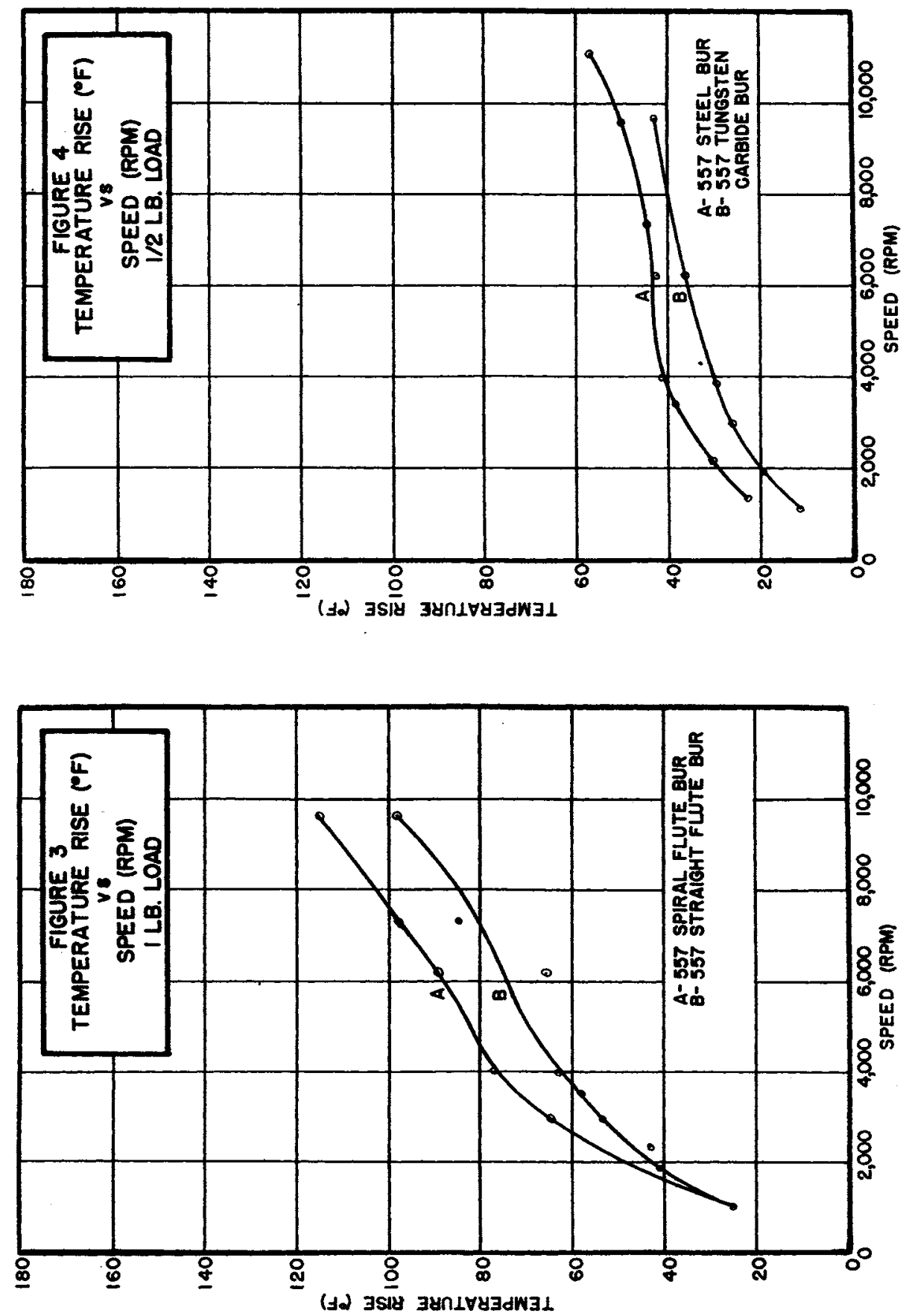
room temperature. From other studies to be reported later, it has been determined that the total temperature rise on extracted teeth measured as described here, is not significantly different from that obtained from teeth in the mouth. ${ }^{3}$

The effect of a change in bur size on temperature rise is demonstrated in Fig. 2, when using a 11/2 pound load. The complete curve for the No. 559 bur was not obtained, but the trend of the curves was established by the use of the No. 557 bur at speeds between approximately 1,000 and 10,000 r.p.m. It is observed that the temperature rise of the smaller No. 557 bur is lower throughout the range tested than that of the No. 559 bur. A pronounced increase in temperature is observed as the speed is increased over the entire range tested.

The difference in the effect of straight and spiral blade or flute design as it influences temperature rise has been studied for the No. 557 bur. The temperature rises developed by the spiral flutted bur and the straight fluted bur are shown in Fig. 3, with a 1 pound load applied. The temperature rise recorded for the spiral bur was higher than that recorded for the straight flutted bur for each speed studied. It has been observed that the spiral grooved bur has greater cutting efficiency than those with straight grooves ${ }^{4}$, therefore under the same conditions of operation, more material will be removed per unit time with the spiral grooved bur than with a comparable straight grooved bur. In this case, the bur which operated most efficiently also developed a higher temperature rise in the tooth.

The temperature rise developed by the No. 557 tungsten carbide bur as compared to a similar steel bur is shown in Figs. 4 and 5 when operating at $1 / 2$ and 1 pound loads, respectively, throughout the speed range from 1,000 to 10,000 r.p.m. The tungsten carbide bur consistently showed a slightly lower temperature rise than the steel bur when operated under comparable conditions. In another study it has been shown that the cutting efficiency of the No. 557 steel bur is similar to that of the No. 557 tungsten carbide bur. ${ }^{4}$ It is possible that the carbide bur develops a little lower temperature rise than the steel bur because of its tendency to resist dulling. Iimited data have been accumulated which would indicate this possibility.

The temperature rise developed for four constant speeds is plotted against applied load in Fig. 6. At the speeds examined it may be observed that an increase in load is accompanied by approximately a linear increase in temperature rise. At slow speeds of operation the temperature rise is approximately doubled as the load is increased from $1 / 2$ to 2 pounds. At higher speeds the rate of temperature rise is even greater as the load is increased. This would support the belief of some operators that relatively light pressures and/or slow speeds are most desirable.

In Fig. 7 the temperature rise is plotted against instrument speed for four loads. The No. 557 steel bur was used for each of these tests. It is interesting to note that as the speed is initially increased the temperature rise is rapid. As the speed is further increased the temperature rise increments become smaller for a short range and then increase as the speed increases to the upper limit of 11,000 r.p.m. It appears from these results that when operating with light pressures, the temperature rise is not excessive, as compared to heavier pressures, even when high speeds are employed. 

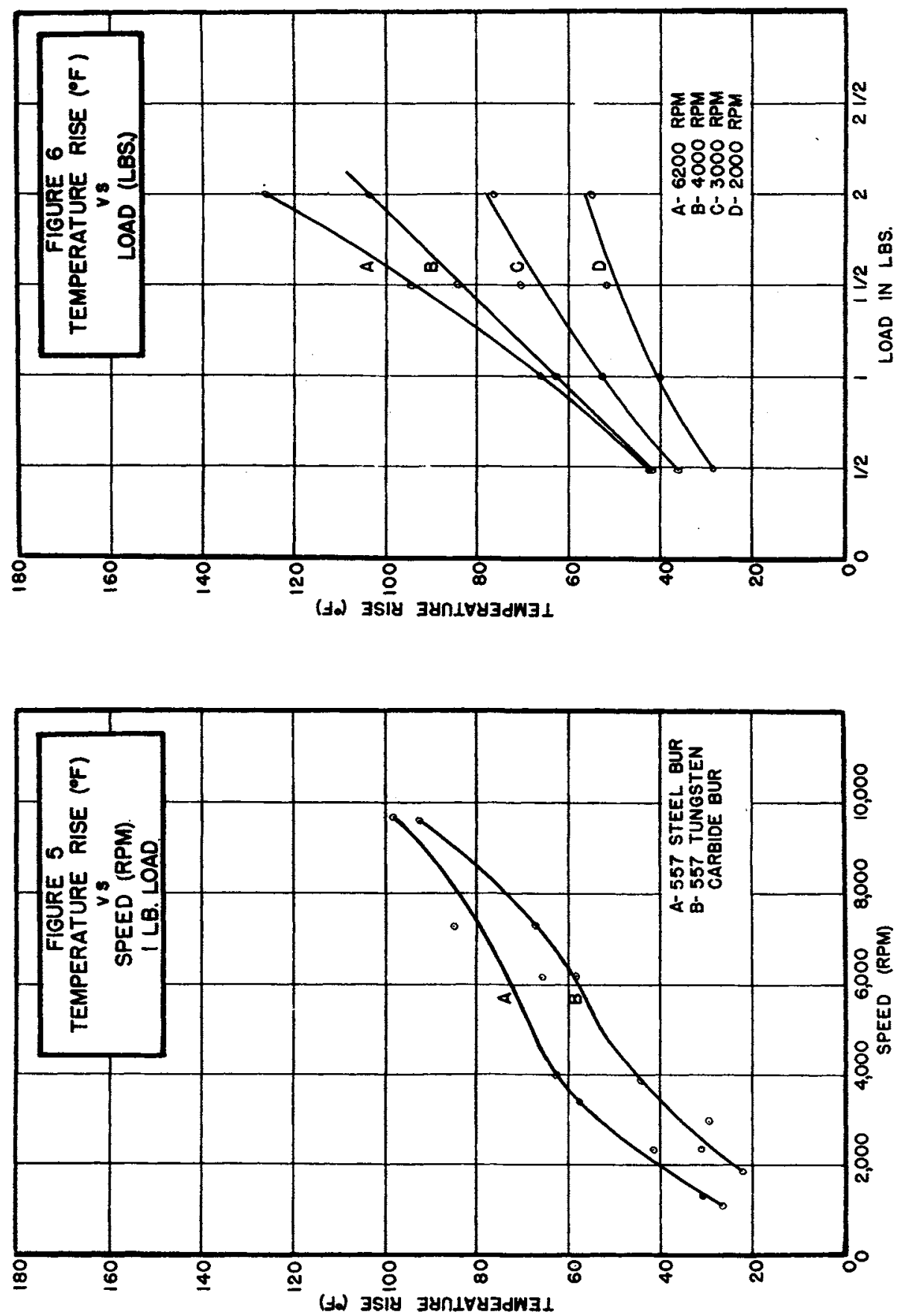
In Fig. 8 is presented a composite three dimensional graph formed by plotting the data contained in Figs. 6 and 7. In this graph, the effect of the two variables, speed and load, upon the temperature rise may be seen.* The maximum temperature rise developed by the two pound load and a speed of approximately 11,000 r.p.m. was not completely established. Temperature rises of more than $160^{\circ} \mathrm{F}$. were recorded, however, at these operating conditions, but further studies are needed to determine with certainty the peak temperatures obtainable.

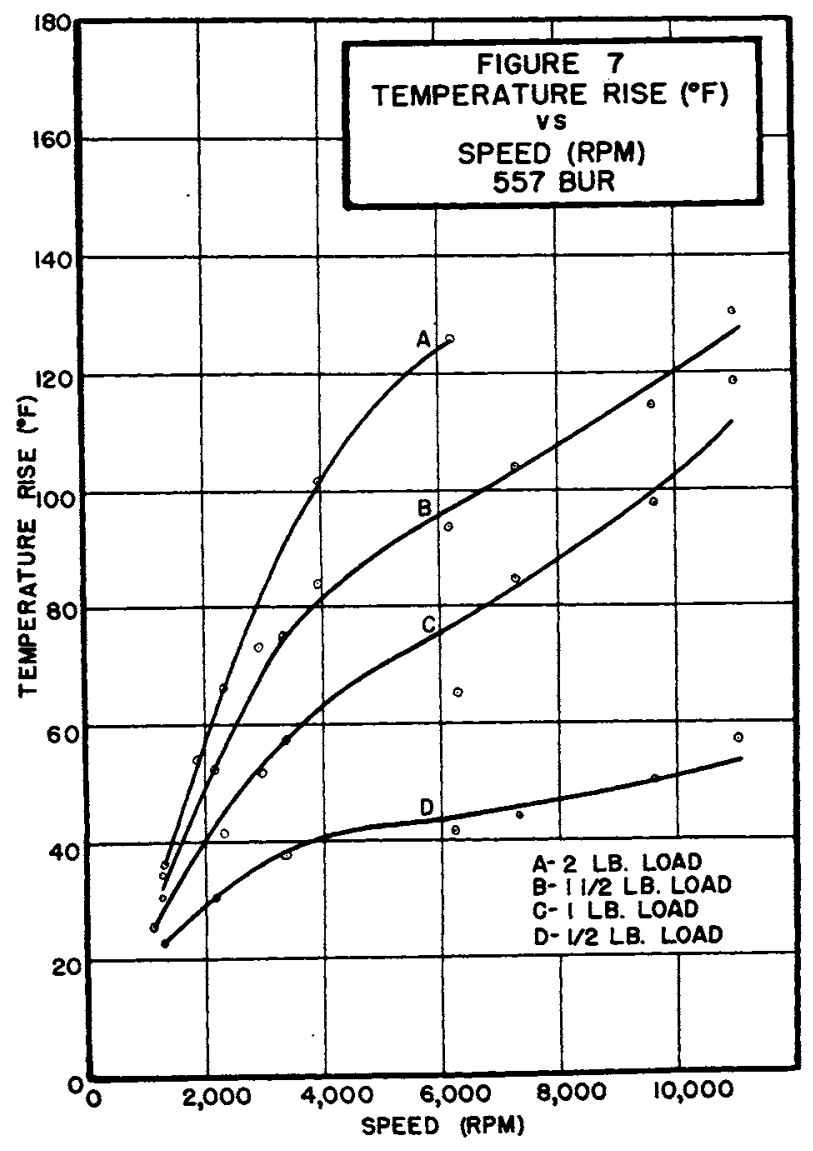

CONCLUSTONS

1. Results have been presented which indicate the temperature rise within the tooth which may be expected when the fissure type bur is used at speeds between approximately 1,100 and 11,000 r.p.m.

2. From the results obtained it appears that near the heat source, or cutting instrument, the maximum temperature rise is developed within ten seconds after the operation begins.

3. The size of the cutting instrument has an influence on the temperature rise observed, and the smaller the bur used the lower the temperature rise. 


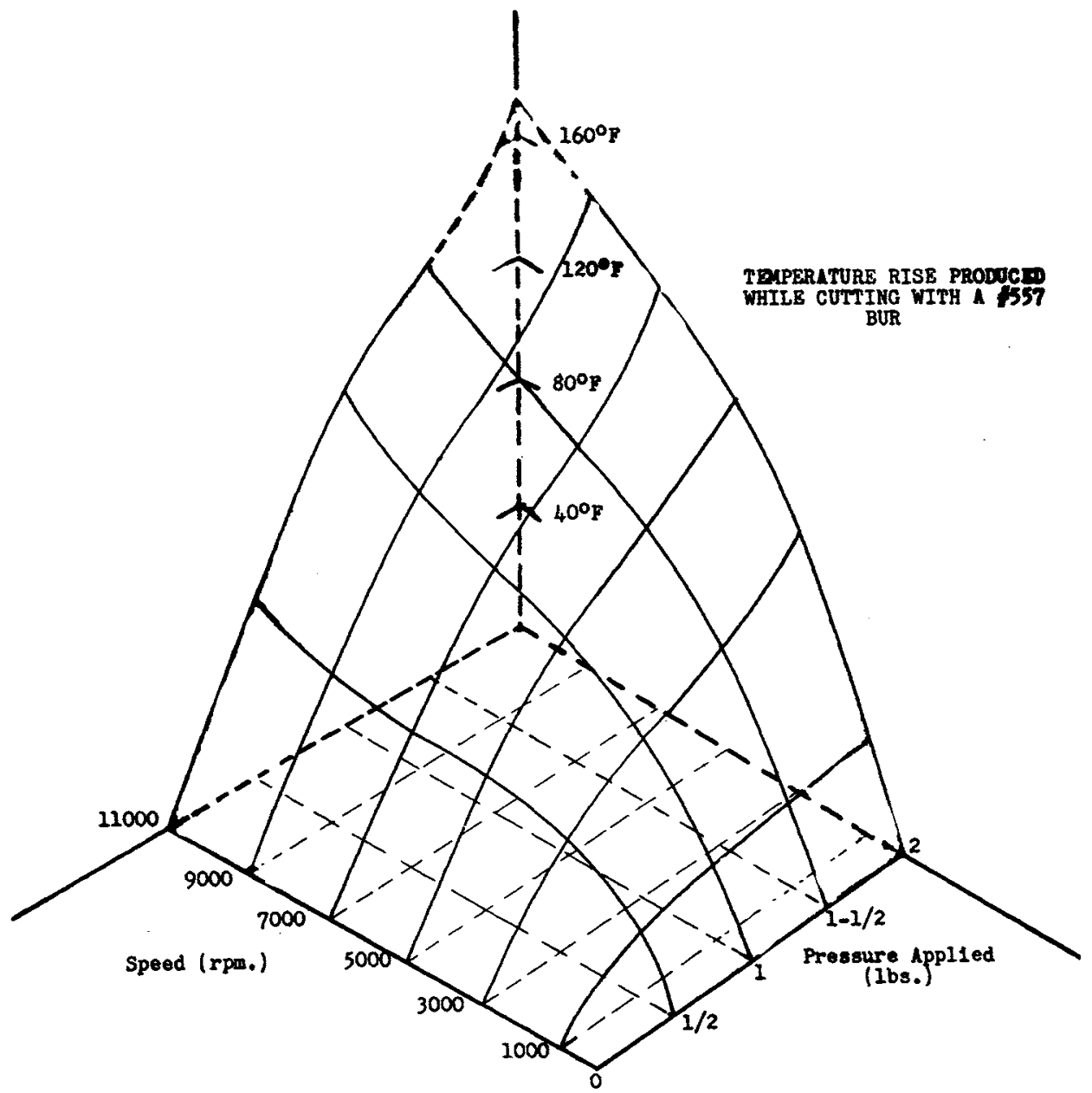

Fig. 8.*

4. The spiral fluted burs studied developed a greater temperature rise than the straight fluted burs.

5. Tungsten carbide burs were shown to produce a lower temperature rise than comparable steel burs operated under the same conditions.

6. Both the increase in pressure and increase in speed of operation were observed to have a pronounced influence on increasing the temperature rise developed in the tooth by all eutting instruments tested.

\section{REFERENCES}

1. Walsh, J. P., and Symmons, H. F.: A Comparison of Heat Produced and Mechanical Efficiency of Diamond Instruments, Stones, and Burs at 3,000 and 60,000 r.p.m., New Zeoland D. J. 45: 28, 1949.

2. Peyton, F. A., and Vaughn, R. C.: Thermal Changes Developed During the Cutting of Tooth Tissue, Fortn. Review of Chi. D. Soc. 20: 9-23, Dec. 15, 1950.

3. Panzer, M.: Unpublished thesis from the University of Michigan Sehool of Dentistry, 1951. 4. Henry, E. E., and Peyton, F. A.: A Study of the Cutting Efficiency of Dental Burs for the Straight Handpieces. To be published.

*The authors acknowledge the assistance of Mr. E. E. Henry for the preparation of 